\title{
Evolution of Magnetic Ring Designs for Phase Plates
}

\author{
C J Edgcombe ${ }^{1}$
}

${ }^{1}$ TFM Group, Dept of Physics, University of Cambridge, CB3 0HE, UK (email: cje1@cam.ac.uk)

Ferromagnetic rings were proposed as phase plates both by the Tonomura group in 1983 [1] and independently in 2009 [2]. They offer the benefits of very low interception of scattered electrons and phase change independent of beam energy. For a phase change of $\pi / 2$, a ring of cobalt $20 \mathrm{~nm}$ thick need only be about $30 \mathrm{~nm}$ wide radially, within an aperture of diameter 50 micrometers. Characterisation of thin-film rings of cobalt has demonstrated their two stable states, one of which consists of the continuous loop or vortex of flux that is desirable for use as a phase plate [3].

A problem with the use of a magnetised ring in a TEM column is that the flux vortex has to remain continuous in the presence of the local axial (or out-of-plane) field. At the objective aperture position, this axial field can be $1 \mathrm{~T}$ or more. First simulations suggested that a cobalt ring of suitable dimensions might be able to maintain a vortex in field of this size. However, a test showed that the loop was destroyed by a field between 0.3 and $0.5 \mathrm{~T}$. When the grain structure of the ring was included in the simulation, the maximum calculated field decreased to a value similar to that observed.

There are other known disadvantages to placing a phase plate at the objective aperture [4]. To avoid these, methods have been developed (for example [5]) to place the plate at a conjugate position, further from the maximum field of the standard objective lens. Another method is to operate in Lorentz mode, turning off the main objective and using the selected-area position as the back focal plane of the Lorentz lens. Although the uncorrected aberrations in the Lorentz mode can be large, they can be corrected by existing methods [6]. Each of these techniques enables the plate to operate in a much lower axial field than that at the objective aperture.

The large reduction in out-of-plane field that the plate must tolerate removes a major constraint on the design of a magnetic-ring plate. It is no longer necessary to use a material with the highest possible saturation magnetization $\left(\mathrm{M}_{\mathrm{s}}\right)$. This has the benefit that the width-to-thickness ratio for the ring can be increased. The flux needed in the ring to provide a suitable phase change is approximately $10^{-15} \mathrm{~Wb}$. This flux is the product of the cross-sectional area of the ring and the $M_{s}$ of the ring material, so if $M_{s}$ is decreased, the cross-section must increase. This increase can be obtained by extending the width of the ring in preference to its thickness. When cobalt is used, a minimum thickness of $20 \mathrm{~nm}$ is needed for the magnetisation to lie in-plane. This restricts the ring width for a phase change of $\pi / 2$ to about $30 \mathrm{~nm}$. However, other materials of lower $\mathrm{M}_{\mathrm{s}}$ may be able to remain magnetised in-plane at smaller thicknesses than is possible for cobalt, enabling the width to be extended further.

An increase in the ratio of width to thickness provides two benefits. First, it raises the shape anisotropy, or energy penalty for flux to escape from the plane of the ring. This further constrains the flux to remain in-plane, thereby increasing the resistance of the vortex state to axial field. Secondly, it may be usable to reduce the effect of white haloes or stripes that appear around large objects when some Zernike phase plates are used to image weak phase objects [7]. 
In the simplest type of Zernike plate, the phase shift changes abruptly from zero to a fixed value, typically $\pi / 2$, at spatial frequency $q=q_{0}=2 \pi r_{2} / \lambda f$, where $2 r_{2}$ is the diameter of the beam hole, $\lambda$ is the electron wavelength, and $\mathrm{f}$ is the focal length of the lens. Calculations show that haloes and ringing occur around circular phase objects for which $B>1$, where $B=q_{0} b$ and $2 b$ is the object diameter [8] (Fig. 1). However, one can imagine the $\pi / 2$ phase change to be distributed instead over a range of radii, outside which it remains constant. Fig. 2 shows the behaviour when the phase change increases linearly from zero at the axis to $\pi / 2$ at spatial frequency $\mathrm{q}_{0}$ and then remains at $\pi / 2$ for greater $\mathrm{q}$. The effect of distributing the phase change in this way is that the ringing is eliminated and the object thickness is modelled more fully. If magnetic material is deposited as a wide ring with constant thickness, the phase change it induces will vary linearly with radius. Hence by suitable choice of dimensions and use of distributed magnetic material, it may be possible to replace the abrupt jump in phase of the simple Zernike plate by a smoother variation in phase, and so to increase the radii at which phase objects are accurately modelled.

\section{References}

[1] N Osakabe et al, patent application JP58-112718, June 1983.

[2] C Edgcombe, EMAG 2009, J Physics Conference Series 241, p. 012005. doi: 10.1088/17426596/241/1/012005.

[3] C Edgcombe, A. Ionescu, J. C. Loudon, A. M. Blackburn, H. Kurebayashi and C.H.W. Barnes, Ultramicroscopy 120 (2012) p. 78; doi:10.1016/j.ultramic.2012.06.011 .

[4] R Glaeser, Review of Scientific Instruments 84 (2013), p. 111101; doi: 10.1063/1.4830355 .

[5] B Barton et al, Ultramicroscopy 111 (2011) p. 1696; doi:10.1016/j.ultramic.2011.09.007 .

[6] B Freitag, M Bischoff, H Mueller, P Hartel and H S von Harrach, Microscopy and Microanalysis, 15 (Suppl. 2)(2009) p. 184.

[7] R Danev, R M Glaeser, K Nagayama, Ultramicroscopy 109 (2009) p. 312.

[8] C Edgcombe, Ultramicroscopy 136 (2014) p. 154, doi: 10.1016/j.ultramic.2013.09.004 .
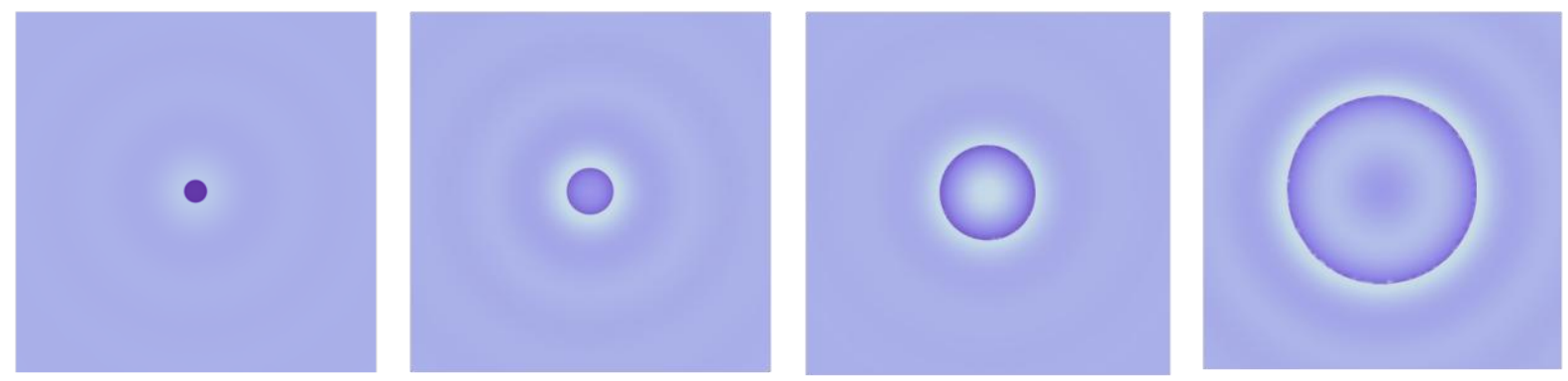

Fig. 1 Images simulated for $\pi / 2$ plate with step cut-on at frequency $\mathrm{q}_{0}$ and object sizes $\mathrm{B}=1,2,4$ and 8 .
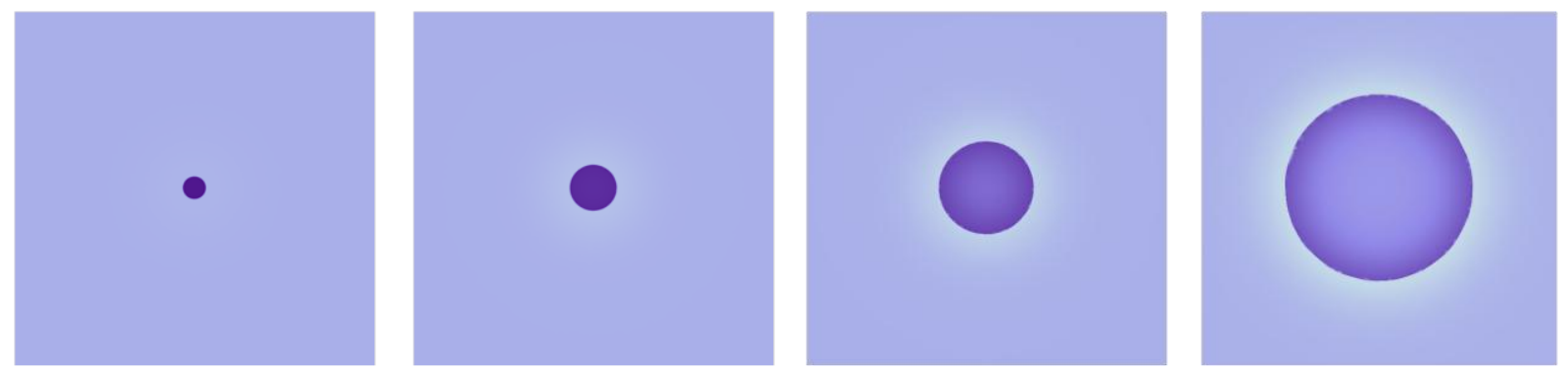

Fig. 2 Images simulated for $\pi / 2$ plate with phase ramp from $\mathrm{q}=0$ to $\mathrm{q}_{0}$ and object sizes $\mathrm{B}=1,2,4$ and 8 . 